\title{
Steroids in severe COVID-19 patients: A retrospective analysis on the first pandemics in Lombardy
}

\author{
Beatrice Vergnano, ${ }^{1}$ Serena Calcinati, ${ }^{1}$ Davide Signori, ${ }^{1,2}$ Annalisa Benini, ${ }^{1}$ Maria Rosa Pozzi, ${ }^{5}$ \\ Luisa Verga, ${ }^{4}$ Jonata Pizzagalli, ${ }^{1}$ Paolo Bonfanti, ${ }^{2,3}$ Giacomo Bellani, ${ }^{1,2}$ Giuseppe Foti ${ }^{1,2}$ \\ ${ }^{1}$ Department of Anesthesia and Intensive Care Medicine, San Gerardo Hospital, Monza; ${ }^{2}$ Department of \\ Medicine and Surgery, University of Milano-Bicocca, Milano; ${ }^{3}$ Clinic of Infectious Diseases; ${ }^{4}$ Medical Area \\ Department, Hematology Division; ${ }^{5}$ Medical Area Department, Rheumatology Unit, San Gerardo Hospital, \\ Monza, Italy
}

\begin{abstract}
The pathogenesis of COVID-19 appears to be characterized by a dysregulated immune response. During the first pandemic wave in Lombardy, we started to administer glucocorticoids to some patients with severe respiratory failure requiring support with Continuous Positive Airway Pressure (CPAP) therapy. We retrospectively collected data to identify the effect of glucocorticoids in this COVID-19 particular population. With a multidisciplinary consensus, we administered to selected patients with severe COVID-19 disease $\left(\mathrm{PaO}_{2} / \mathrm{FiO}_{2} 159 \pm 71 \mathrm{mmHg}\right) 0,91$
\end{abstract}

\author{
Correspondence: Davide Signori, Department of Anesthesia and \\ Intensive Care Medicine, San Gerardo Hospital, Via G. B. Pergolesi 33, \\ 20900 Monza, Italy. \\ Tel.: +39.3480939103 \\ E-mail: d.signori24@gmail.com
}

Key words: COVID-19; steroids; acute respiratory failure; CPAP; inflammation.

Acknowledgments: The authors would like to thank Pietro Pioltelli,4 Michela Bombino,1 Stefano Isgrò,1 Guglielmo Marco Migliorino,3 Alessandro Soria.3

Conflict of interest: The authors declare no conflict of interest.

Availability of data and materials: All data generated or analyzed during this study are included in this published article.

Ethics approval and consent to participate: For this observational, single center study data were collected in a local online registry as part of the STORM study (Spallanzani Institute approval number 84/2020; NCT04424992). Patients' consent was waived in the context of the authorizations guaranteed by Article 89 of the GDPR EU Regulation 2016/679.

Informed consent: Patients' consent was waived.

Received for publication: 3 June 2021.

Revision received: 21 July 2021.

Accepted for publication: 16 August 2021.

This work is licensed under a Creative Commons Attribution 4.0 License (by-nc 4.0).

${ }^{\circ}$ Copyright: the Author(s), 2021

Licensee PAGEPress, Italy

Emergency Care Journal 2021; 17:9888

doi:10.4081/ecj.2021.9888 $\mathrm{mg} / \mathrm{kg} /$ die of methylprednisolone equivalent dose after a median of 8 days of hospitalization. In our study we compared 57 patients from the steroid group with 123 from the control group: the event of invasive mechanical ventilation or death was reduced by $43 \%$ between steroid group and control group (19.3\% vs. $34.1 \%$ respectively, $\mathrm{p}=0.001$ ) and mortality was reduced by about $31 \%$ between steroid and usual care alone (15.8 \% vs. $22.8 \%$ respectively, $\mathrm{p}=0.011)$. Corticosteroids in selected COVID-19 patients may have a relevant impact on outcome, better profiling of the heterogeneity of this disease may be essential to guarantee the best treatment choices.

\section{Introduction}

The pathogenesis of COVID-19 appears to be characterized by a dysregulated immune response which causes systemic and pulmonary inflammation, along with endothelial injury, hypercoagulability and thrombosis..$^{1-3}$ Recent findings support the use of glucocorticoids to modulate the immune response aiming to decrease the effects of hyperinflammation..$^{2-9}$ The beneficial effect of glucocorticoid therapy has been described in ICU patients, while the evidence for patients requiring ventilatory support outside and/or before the ICU is not univocal, also due to the lack of data in this population.

The Randomized Evaluation of COVID-19 Therapy (RECOVERY) trial ${ }^{5}$ found the greater benefit from steroids in invasive ventilated patients and, to a lesser degree, among those receiving oxygen without invasive Mechanical Ventilation (MV); unfortunately, a stratification for different respiratory support levels between simple oxygen supplementation and ventilatory support systems was not reported in that paper.

The following WHO meta-analysis, ${ }^{6}$ reviewing 1703 critically ill patients, confirmed the beneficial effect of glucocorticoids administration; interestingly the strongest signal was founded among non-invasively ventilated patients, which accounted just for $8.5 \%$ of the cases (144 patients).

Many retrospective cohort and case-series studies have yielded conflicting results on the efficacy of glucocorticoids for the treatment of COVID-19. Since different severity of illness and different steroid dosage and timing of administration where reported, ${ }^{10-14}$ better profiling and targeting of COVID-19 patients is desired; thus, several RCTs are ongoing. ${ }^{15-17}$

During the first pandemic wave, in our third level hospital in Lombardy, on the basis of a multidisciplinary collaboration between intensivists, hematologists, rheumatologists and infectivologists we started to immunomodulate some patients with severe respiratory failure, still not requiring MV. 
In this paper we report the results of our experience during the first wave of pandemics.

\section{Materials and Methods}

From February 2020, in San Gerardo Hospital, a large tertiary teaching hospital, based upon increasing pathophysiological knowledges and clinical early experience, systemic glucocorticoids were administered to selected patients with severe COVID-19 who were not mechanically ventilated, opening the opportunity to assess their contribution to manage this disease.

All patients have been managed using the best supportive treatment following the indications available at the time at the judgment of the physician.

Relying on early experience and the limited but increasing physio-pathological knowledge, our multidisciplinary group began prescribing systemic glucocorticoids to patients with COVID-19 referred to MET. Each case was discussed daily and therapeutic strategy choice was made taking into account medical history and clinical course: steroid therapy was usually administered to patients that didn't show improvement after about one week of standard therapy. No specific algorithm for steroid administration was produced at the time since they were relatively contraindicated. ${ }^{14,18,19}$

We took advantage of this change in practice to investigate the association between glucocorticoids use and mortality and the rate of MV in hospitalized COVID-19 patients.

We retrospectively reviewed medical records of COVID-19 patients referred to MET (Medical Emergency Team) who received Continuous Positive Airway Pressure Therapy

(CPAP)support in the ED and/or medical ward.

For this observational, single center study data were collected in a local online registry as part of the STORM study (Spallanzani Institute approval number 84/2020; NCT04424992). Patients' consent was waived.

\section{Inclusion criteria}

For this observational, retrospective, single center study we considered eligible adult patients (age $\geq 18$ years) admitted to San Gerardo hospital, between February $27^{\text {th }}$ and April $30^{\text {th }} 2020$, confirmed to be positive for SARS-CoV-2 by reverse transcriptionpolymerase chain reaction of pooled nasal and pharyngeal swabs.

Only patients referred to the MET for more than 24 hours by the ED or medical wards with a confirmed diagnosis of COVID19-related severe pneumonia were included.

\section{Exclusion criteria}

We excluded patients treated with steroids for less than 48 hours before death or start of MV; equally, those treated for other reasons or with chronic steroid therapy were considered not eligible.

Moreover, patients were excluded if they were transferred from an out-of-system hospital without complete medical records, if considered moribund on the basis of clinical judgment or died within 24 hours since the presentation to the ED.

\section{Analysis focus}

Since steroid therapy was administered after a few days of hospitalization, to adjust our results for immortal time bias (i.e. patients must survive long enough to receive the intervention of interest) leading to a potential incorrect overestimation of a positive treatment effect, we excluded from the primary analysis those who died, were intubated or de-escalated CPAP before the median time of steroid beginning since the hospital admission. Data and analysis regarding the whole population that met inclusion and exclusion criteria is also reported in this manuscript.

\section{Data collection}

Data were collected from electronic patient records and entered, anonymously, into a securely stored database.

We collected demographics, Charlson Comorbidity Index (CCI) as a performance status indicator, Sequential Organ Failure Assessment (SOFA) score, timing of symptoms onset, hospital admission and MET referral.

We focused on five significant events in the course of the disease: hospital admission, onset of $\mathrm{O} 2$ administration, onset of CPAP support, initiation of steroid administration (if available) and the final multiple event defined as de-escalation of $\mathrm{O} 2$ support (to $\mathrm{FiO}_{2}<40 \%$, no more need of CPAP support and $\mathrm{PaO}_{2} / \mathrm{FiO}_{2}>300$ ) OR tracheal intubation and ICU admission OR withdrawal of treatment.

\section{Outcomes}

The primary outcome was a composite of in-hospital mortality or MV.

Secondary outcome was in-hospital all causes mortality rate.

The independent variable of interest was treatment with glucocorticoids.

\section{Statistical analysis}

Statistical analysis and graphs were performed with IBM SPSS Statistic v. 27.

Continuous variables are summarized as mean values with standard deviations or median values and interquartile range for normal and non-normal distribution respectively. Categorical variables were summarized as counts and percentages.

Population characteristic comparison between the control group and the steroid group was performed with an independentsample T-test for continuous variables and Chi-Square statistic for categorical variables.

Paired sample T-test was performed for comparison of variation of CRP (between hospital admission and initiation of steroids) and of $\mathrm{PaO}_{2} / \mathrm{FiO}_{2}$ (between onset of CPAP support and initiation of steroids).

For the primary composite outcome of invasive MV or death a multinomial logistic regression (with multivariate analysis for steroid therapy, age, $\mathrm{CRP}$ and $\mathrm{PaO}_{2} / \mathrm{FiO}_{2}$, CCI and SOFA score) was used to estimate the odds ratio.

For the secondary outcome (in hospital mortality) we performed a multinomial logistic regression (with multivariate analysis for steroid therapy, age, CRP and $\mathrm{PaO}_{2} / \mathrm{FiO}_{2}$ ) due to the low number of exitus.

Interaction between steroid therapy and age, CRP, $\mathrm{PaO}_{2} / \mathrm{FiO}_{2}$ and CCI were also analyzed in the multivariate analysis.

\section{Results}

\section{Population characteristic}

In our hospital between February $27^{\text {th }}$ and April $30^{\text {th }}, 2020$ we admitted 1026 COVID-19 patients and more than an half were reviewed by the MET at least once; 471 patients were strictly followed by the MET service for at least 2 days or more receiving helmet CPAP in a non-intensive care setting. Thirty patients were 
excluded because they were referred to the MET for less than 24 hours. 70 patients met other exclusion criteria. Hence the analysis included 371 patients (305 in control group, 66 in steroid group). Whole population characteristics are summarized in Supplemental Table 2. Patients from control group were younger (65vs. 69 years old) and had generally more severe systemic disease (SOFA $3.9 \mathrm{vs}$. 3.4) compared to the steroid group. However, no difference in respiratory failure severity categorized as $\mathrm{PaO}_{2} / \mathrm{FiO}_{2}$, neither in comorbidity (Charlson Comorbidity Index), neither in grade of inflammation (C-Reactive Protein) were evident. CPAP were started after a median of 1 day after hospital admission in both groups, however on average the steroid group received this support 1 day later $(\mathrm{p}=0.021$; Figure 1$)$.

Using the first COVID-19 patient's date of access to our hospital as the reference, the steroid group was admitted 7 days later (95\% CI: 4-10) than the usual care group (Figure 2). The time of hospital admission does not modify the outcomes in the multivariate analysis.

Considering the steroid group, the mean administered steroid dose was $0.91(\mathrm{SD} \pm 0,26) \mathrm{mg} / \mathrm{kg} /$ die of prednisone equivalent dose (largely methylprednisolone, exceptionally dexamethasone). At steroid therapy beginning these patients had $\mathrm{PaO}_{2} / \mathrm{FiO}_{2}$ of $159 \pm 71$ $\mathrm{mmHg}$. The mean duration of steroid therapy was 14.5 days (IQR 9-30).

The median number of days between hospital admission and steroid administration was 8 (IQR 5-14). To adjust our results for immortal time bias, we excluded patients who met the composite outcome before that date. After applying this filter, the statistical analysis was performed on 180 patients (123 control and 57 steroid, Figure 1); population characteristics are summarized in Table 1.

Age was significantly higher by 4.5 years (95\% CI: $1.1-8.0)$ in the steroid group. CRP at hospital admission was $2.4 \mathrm{mg} / \mathrm{L}$ higher in the steroid group ( $\mathrm{p}=0.046$ ), while the same values at first CPAP trial did not reach the level of significance $(p=0.051)$. No difference in comorbidity, illness severity, days of symptom before hospital access and days between admission and first CPAP trial were found between the two groups.

\section{Primary outcome}

The event of invasive MV or death was significantly lower in the steroid group than in the usual care group according to the multivariate analysis for steroid therapy, age, CRP and $\mathrm{PaO}_{2} / \mathrm{FiO}_{2}$ (OR 0.196; 95\% CI 0.078-0.497; Table 2).

The event of invasive MV or death was $43 \%$ lower in the steroid group compared to control group (19.3\% vs. $34.1 \%$ respectively, $\mathrm{p}=0.001$ ).

Age, CRP recorded at hospital admission and SOFA score were independent predictors of bad outcome, while no effect of $\mathrm{CCI}$ and $\mathrm{PaO}_{2} / \mathrm{FiO}_{2}$ at first CPAP trial was evident.

The interactions between steroid therapy and age, CRP and $\mathrm{PaO}_{2} / \mathrm{FiO}_{2}$ on primary outcome are graphically summarized in Figure 3. The event MV or death: i) was more frequent in older patients who did not undergo steroid treatment $(\mathrm{p}<0.001)$ while no relation was seen in those with steroid therapy $(\mathrm{p}=0.378$; Panel A); ii) was more likely in patients with higher CRPs with no steroid therapy ( $p=0.021)$ while no association was seen in steroid therapy group ( $\mathrm{p}=0.236$; Panel $\mathrm{B})$; iii) had no statistically significant

Table 1. Population baseline comparison between usual care alone and usual care + steroid.

\begin{tabular}{|c|c|c|c|}
\hline & $\begin{array}{l}\text { Treatment } \\
\text { Control group } \\
(\mathrm{N}=123)\end{array}$ & $\begin{array}{l}\text { Steroids group } \\
\qquad(\mathrm{N}=57)\end{array}$ & P-value \\
\hline Age - Years & $64 \pm 11$ & $68 \pm 10$ & $0.010^{*}$ \\
\hline Female - no. (\%) & $29(24)$ & $17(30)$ & 0.371 \\
\hline Days of symptoms before admission & $7 \pm 6$ & $7 \pm 5$ & 0.438 \\
\hline Days between admission and CPAP therapy & $3 \pm 3$ & $3 \pm 4$ & 0.355 \\
\hline $\mathrm{CCI}$ & $3.5 \pm 2.0$ & $3.9 \pm 1.3$ & 0.170 \\
\hline SOFA score & $3.3 \pm 1.5$ & $3.4 \pm 1.2$ & 0.847 \\
\hline $\mathrm{CRP} £-\mathrm{mg} / \mathrm{L}$ & $9.7 \pm 6.8$ & $12.1 \pm 8.3$ & $0.046^{*}$ \\
\hline $\mathrm{CRP} \dagger-\mathrm{mg} / \mathrm{L}$ & $11.6 \pm 7.3$ & $14.0 \pm 7.5$ & 0.051 \\
\hline $\mathrm{PaO}_{2} / \mathrm{FiO}_{2} \dagger-\mathrm{mmHg}$ & $247 \pm 116$ & $217 \pm 114$ & 0.114 \\
\hline Admission time since first patient entrance - days & $23 \pm 9$ & $30 \pm 10$ & $<0.001^{*}$ \\
\hline
\end{tabular}

CCI: Charlson Comorbidity Index; SOFA: Sequential Organ Failure Assessment; CRP: C-Reactive protein; *: $p<0.05$; £: Value recorded at hospital admission; $\dagger$ : Value recorded at first CPAP trial.

Table 2. Multivariate analysis for primary outcome and relative Odds Ratio.

\begin{tabular}{|c|c|}
\hline $\begin{array}{l}\text { Effect of steroid treatment - Main results } \\
\text { Primary outcome: mechanical ventilation or death }\end{array}$ & Adjusted Odds Ratio (95\% CI) \\
\hline Steroid therapy & $0.198(0.073-0.536)^{*}$ \\
\hline Age - years & $1.168(1.010-1.128)^{*}$ \\
\hline $\mathrm{PaO}_{2} / \mathrm{FiO}_{2} \dagger-\mathrm{mmHg}$ & $0.999(0.995-1.003)$ \\
\hline CRP£ - mg/L & $1.069(1.010-1.131)^{*}$ \\
\hline $\mathrm{CCI}$ & $1.130(0.835-1.530)$ \\
\hline SOFA score & $2.006(1.433-2.808)^{*}$ \\
\hline
\end{tabular}

Missed data for primary and secondary outcome: 171 cases of 180 (53/57 in steroid group, 118/123 in standard care alone group). CRP: C-Reactive protein; CCI: Charlson Comorbidity Index; SOFA: Sequential Organ Failure Assessment. *: $\mathrm{p}<0.05$; §: Value recorded at hospital admission; $\uparrow$ : Value recorded at first CPAP trial; £: Value recorded at hospital admission 
relation with $\mathrm{PaO}_{2} / \mathrm{FiO}_{2}$ in both control and steroid groups, even if the higher rates of MV or death in patients with $\mathrm{PaO}_{2} / \mathrm{FiO}_{2}<200$ $\mathrm{mmHg}$ was shown (Panel C).

The interaction between steroid therapy and CCI on primary outcome is graphically summarized in Figure 4. The event MV or death had no statistically significant relation with $\mathrm{PaO}_{2} / \mathrm{FiO}_{2}$ in both control and steroid groups.

\section{Secondary outcome}

In hospital mortality was significantly lower in the steroid group compared with standard care alone group according to the multivariate analysis for steroid therapy, age, $\mathrm{CRP}$ and $\mathrm{PaO}_{2} / \mathrm{FiO}_{2}$ (OR 0.269; 95\% CI 0.097-0.743). Multivariate analysis for the secondary outcome is reported in Appendix Table 1. The inhospital mortality was reduced by about $31 \%$ between steroid and usual care alone $(15.8 \%$ vs. $22.8 \%$ respectively, $\mathrm{p}=0.011)$.
Age and CRP recorded at hospital admission were an independent predictor of mortality, while non effect of $\mathrm{PaO}_{2} / \mathrm{FiO}_{2}$ at first CPAP trial was evident.

\section{Supplemental data}

Other analysis on CRP and $\mathrm{PaO}_{2} / \mathrm{FiO}_{2}$ recorded at steroid therapy administration, as well as data regarding patients aposteriori excluded from analysis, are available in the appendix.

\section{Discussion}

The main findings of this study can be summarized as follows: i) patients with severe COVID-19 respiratory failure needing CPAP support and hospitalized from at least 1 week may have strong benefits from steroid therapy adjusted on body weight in

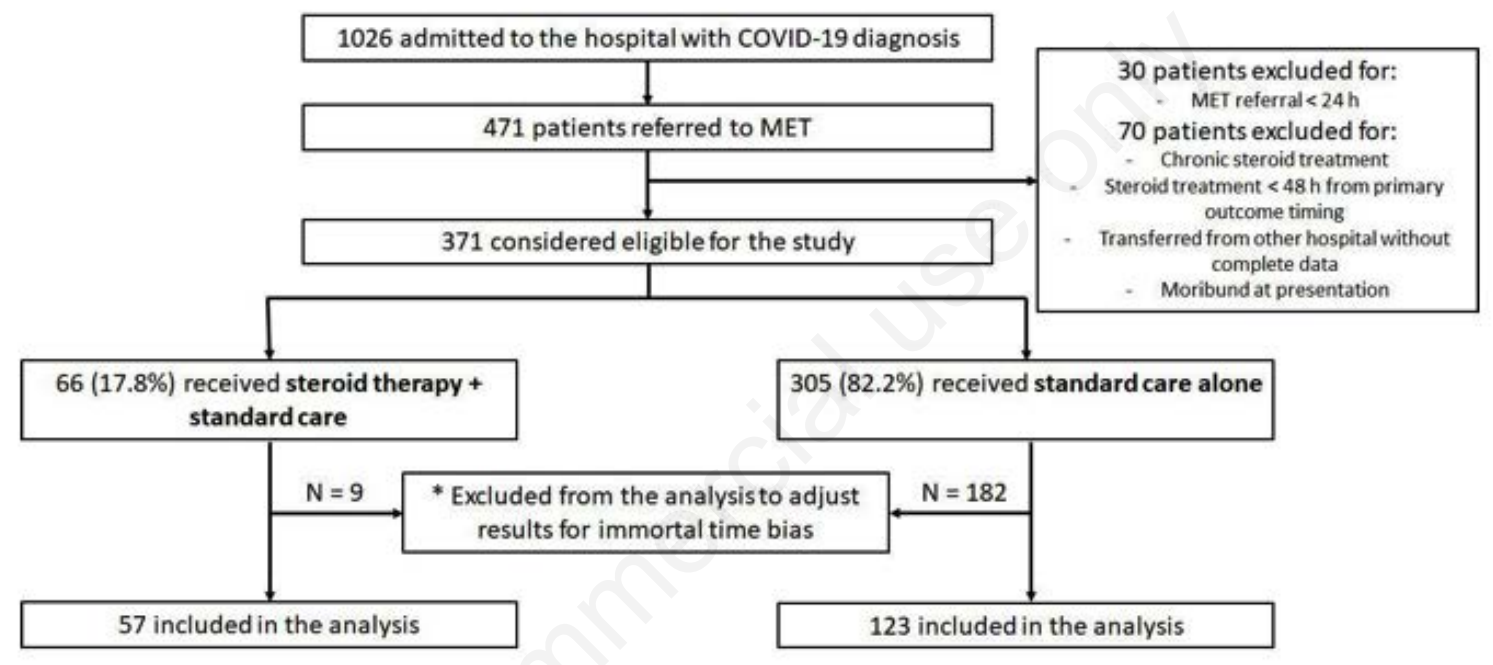

Figure 1. Enrollment, exclusion and patients distribution in the primary analysis. ${ }^{*}$ Since steroid therapy was administered after a median of 8 days of hospitalization, we excluded those who died, were intubated or de-escalated CPAP before 8 days since the admission.

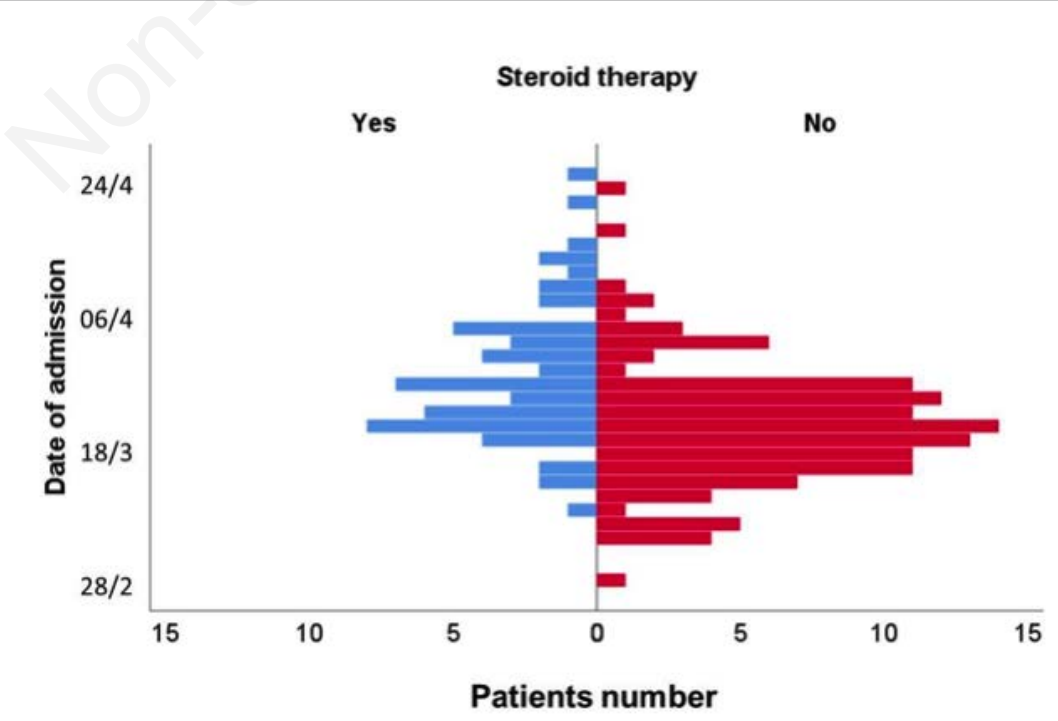

Figure 2. The steroid group vs. usual care group time of hospital admission since first COVID-19 patient admission (February 28th, 2020). an of 8 days of hospitalization, we excluded those who died, were intubated or de-escalated CPAP before 8 days since the admission. 
terms of hospital mortality and rate of MV; ii) steroid therapy seems to mitigate age and CRP effects on mortality and rate of MV.

During the so called first wave in Lombardy, as a consequence of limited ICU resources due to the overwhelming number of patients needing respiratory assistance, many critical patients were treated outside the ICU setting. ${ }^{20,21}$ In our hospital this was facilitated by our experience with MET which was initially introduced to avoid inappropriate ICU admissions and to start prompt intensive treatment. It evolved over the years introducing the possibility of receiving helmet CPAP, amine support and other intensive treatments in non-intensive wards. This organization was discovered precious during the pandemic when crisis management systems and logistic support became essential. ${ }^{22}$

Patients with severe COVID-19 can develop a systemic inflammatory response leading to lung injury and multisystem organ dysfunction. ${ }^{1,3,7,23-25}$ Based on the pathogenesis of COVID19 , approaches that modulate the immune response may have greater impact in the later phases of the disease..$^{5,12,25-27}$ Relying on early experience and the limited but increasing physiopathological knowledge, our multidisciplinary group began prescribing systemic glucocorticoids to patients with COVID-19.

Slightly after, evidences in favor of glucocorticoids rapidly raised and their ability to prevent or mitigate the deleterious effects of inflammatory activation ${ }^{1,7}$ and even to reduce mortality in COVID-19, especially in more severe groups of patients, was clear. $^{5-7}$

RECOVERY trial demonstrated that dexamethasone (6mg daily for 10 days) in hospitalized patients with COVID-19 reduced 28-days mortality, duration of hospitalization and progression to invasive MV. The greater benefit on survival was observed in the invasive ventilated patient subgroup. ${ }^{5}$

Since our study is retrospective, a comparison with RECOVERY trial would not be appropriate, however clear differences in methodological structure are evident. Particularly, in our study dosage was adjusted for the weight (on average 1 $\mathrm{mg} / \mathrm{kg} /$ die of methylprednisolone equivalent dose). It is reasonable that administering the same dosage (6 $\mathrm{mg}$ of dexamethasone) to all the patients without any adjustment could have some limitations. In addition, it has been demonstrated in different clinical settings that rapid clearance and high level of dilution of glucocorticoids
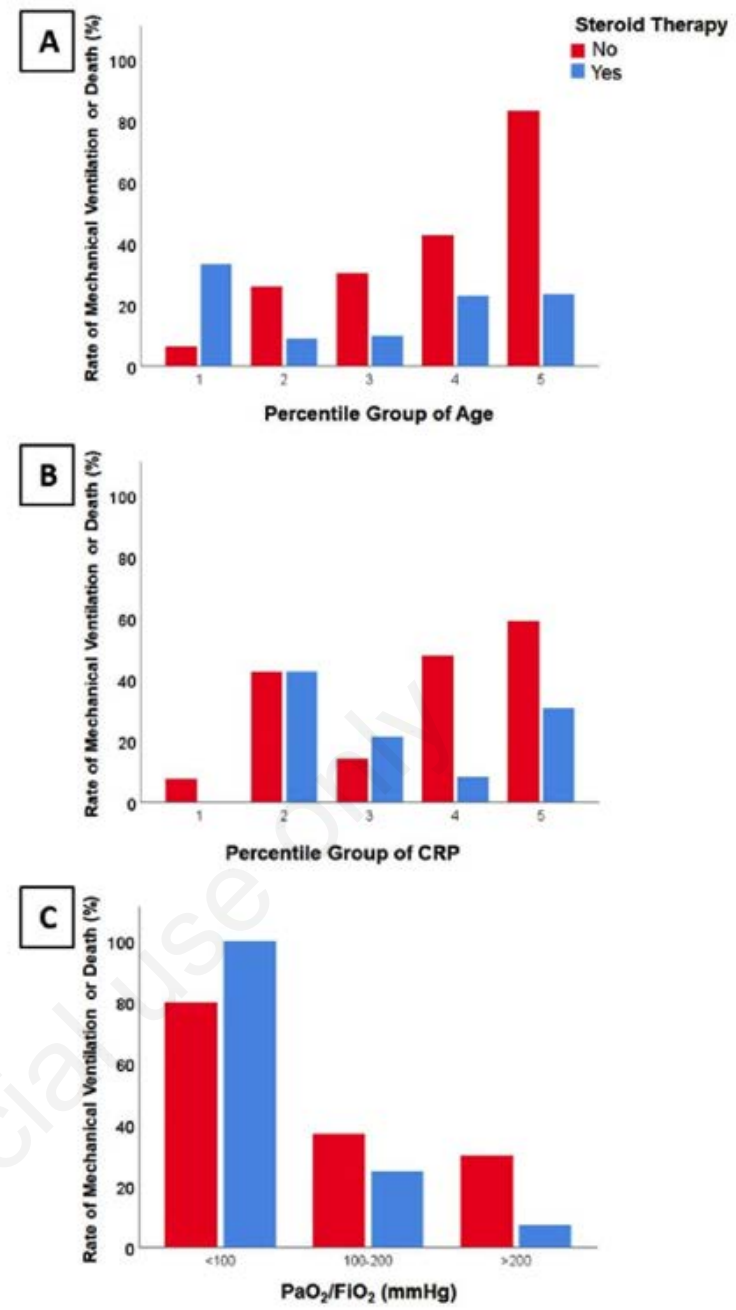

Figure 3. The Steroid vs. control groups rate of mechanical ventilation or death in relation to Age quintiles (Panel A), hospital admission CRP quintiles (Panel $\mathrm{B}$ ), and $\mathrm{PaO}_{2} / \mathrm{FiO}_{2}$ at CPAP positioning main division (Panel C). CRP: C-Reactive Protein.

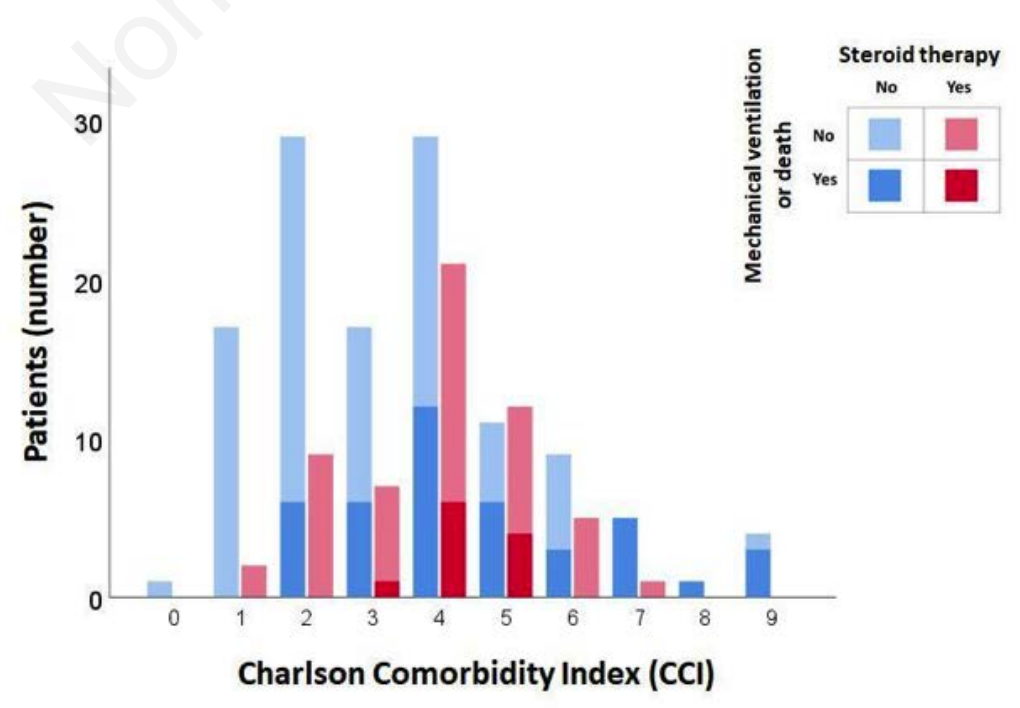

Figure 4. The Steroid vs. control groups patients distribution for CCI score. In vivid colors are highlighted patients who met the composite outcome invasive mechanical ventilation or death. CCI: Charlson Comorbidity Index. 
among obese patients may contribute to persistent inflammation and their suboptimal response to systemic glucocorticoids. ${ }^{28,29}$

Moreover, at the time of steroid administration, our patients were already hospitalized for 8 days, were symptomatic for 15 days on average and had a significant respiratory failure even with the maximization of CPAP assistance ( $\mathrm{PaO} 2 / \mathrm{FiO} 2159 \pm 71$ $\mathrm{mmHg}$ ). In Recovery trial dexamethasone was generally administered in an earlier and milder stage of the disease according to the staging proposed by Siddiqi et al. ${ }^{26}$ and subsequently suggested elsewhere. ${ }^{9,25}$

Indeed, evidence from the RECOVERY also suggests that benefit from corticosteroids is clear only after more than 7 days since symptoms onset, supporting our thesis. Also, no stratification was made for different respiratory support levels between simple oxygen supplementation and ventilatory support systems as helmet CPAP, HFNC or NIMV, even if during the pandemic these systems have been largely used in medical wards ${ }^{20,21}$ consequently to the decreased accessibility of intensive care beds. Finally, authors themself admitted a possible harm of steroid therapy in the subgroup of patients not receiving oxygen support.

Interestingly, our patients requiring CPAP support and hospitalized for slightly more than one week represent a particular sub-group of the disease variety: excluding patients with hyperacute respiratory failure who needed immediate intensive treatment or rapidly died and those who had a favorable evolution, we had to face those who were not improving after 8 days of hospitalization and 6 after CPAP support (15 days from illness onset). Only at this point we considered steroids, and our results on this population are encouraging: steroids were effective in reducing the event $\mathrm{MV}$ or death from 34.1 to $19.3 \%(\mathrm{p}=0.001)$ and mortality from 22.8 to $15.8 \%(\mathrm{p}=0.011)$ despite the steroids group was 4 years older than the control group.

Our results are consistent with those of the RECOVERY trial and, considering the absolute reduction in mortality and MV rate, underline a possible greater benefit in a specific phase and severity of COVID-19.

Moreover, different studies ${ }^{2,8-10,14,25,27,30,31}$ suggest non benefit/possible harm of glucocorticoid administration in nonsevere patients or in too early phases of the disease. This may explain the more beneficial effect on our population.

The WHO meta-analysis ${ }^{7}$ compared the effect of steroids in severe COVID-19 patients, using data from seven randomized clinical trials, along with RECOVERY. The analysis was centered on mechanically ventilated patients $(91,5 \%)$, however 144 critically ill patients $(8.5 \%)$ were not on MV. On those 144 patients, the meta-analysis confirmed a decreased 28-day mortality in patients treated with glucocorticoids and interestingly, the analysis indicated that glucocorticoid use was of greater benefit in critically ill patients who did not undergo invasive $\mathrm{MV}$, in accordance with our results.

The absence of clinical trials on steroid efficacy at the time of the first wave in Italy (with expert consensus expressing relative contraindication $)^{14,18,19,32}$ is the reason why a great number of patients in our study did not receive steroids and allow our comparison.

In our population younger patients $(57 \pm 11$ years old), with lower degree of respiratory failure (even if with $\mathrm{PaO}_{2} / \mathrm{FiO}_{2} 265 \pm 81$ $\mathrm{mmHg}$ with CPAP therapy), low CCI $(2.4 \pm 1.8)$ and CRP $(10.2 \pm 5.5$ $\mathrm{mg} / \mathrm{L}$ at hospital admission) had a good outcome just with supportive therapy. These results also suggest that a better profiling for glucocorticoid eligibility is needed.

Assessing the steroids effect on mechanically ventilated patients is not the core aim of our study since MET role is to triage and treat patients outside the ICU; moreover, we think that complex and conflicting interaction of steroids and ARDS S $^{1,25,30-36}$ combined with COVID-19 pathophysiology, may need specific categorization.

In this study age and CRP recorded at hospital admission are independent predictors of both mortality and composite outcome $\mathrm{MV}$ or death. Interestingly, the interaction between age and CRP with steroids showed that the predicting value of age and CRP is evident only in the control group, while the Steroids outcomes appears not to be influenced by these 2 factors. (Figure 3, Panel A and B). These data are consistent with other studies that already underlined the importance of age and CRP in this disease. More importantly, the rationale for steroids therapy seems to be even stronger as CRP is widely considered a marker of severity of inflammation in COVID-19.37-39

Considering Panel A, the graphic representation suggests that steroids in younger patients may be harmful. However, due to the low number of patients included in the first quintile of age, we retained that a specific analysis on this group may be of poor statistical significance. These data may benefit from further investigations since the harmful effects of steroids in younger patients can't be excluded.

$\mathrm{PaO}_{2} / \mathrm{FiO}_{2}$ at the first CPAP trial seems not to be an independent predictor of bad outcome, however the $95 \% \mathrm{CI}$ in the multivariate analysis for composite outcome and mortality touch the level of significance without reaching it (Table 2). Moreover, the graphical appearance of the relation between $\mathrm{PaO}_{2} / \mathrm{FiO}_{2}$ and $\mathrm{MV}$ or death rate shows a greater incidence of bad outcome in patients with $\mathrm{PaO}_{2} / \mathrm{FiO}_{2}<100 \mathrm{mmHg}$ (Figure 3, Panel C). According to these data, $\mathrm{PaO}_{2} / \mathrm{FiO}_{2}$ at CPAP must be considered a variable influenced by many conditions such as cardiac function or body positioning at the time it was measured; ${ }^{40}$ prone position in awake patients in COVID-19 is associated with better oxygenation even if at this time no benefit of this procedure on outcome is evident.

In our additional analysis on a-posteriori excluded patients, wide population heterogeneity is evident: even if every patient needed CPAP support during the course of the disease, the evolution of the disease followed a different, sometimes opposite journey. Corticosteroids were marginally administered in this subgroup because of the clinical approach we adopted to prescribe this treatment. These patients may benefit or not of immunomodulating therapies; better profiling of this cohort is needed to manage the wide phenotypes variety of COVID-19.

\section{Conclusions}

Steroid therapy seems to be beneficial in severe respiratory failure COVID-19, reducing the mortality and the necessity of MV in our population.

Our results suggest a better profiling of COVID-19 patients for optimal rationales, timings, dosages of steroids since efficacy seems to differ broadly considering age, timing, disease severity and comorbidity.

CRP, age and SOFA are confirmed to be independent prognostic factors in COVID-19 in our population.

\section{References}

1. Arabi YM, Chrousos GP, Meduri GU. The ten reasons why 
corticosteroid therapy reduces mortality in severe COVID-19. Intensive Care Med 2020;46:2067-70.

2. Cano EJ, Fonseca Fuentes X, Corsini Campioli C, et al. Impact of Corticosteroids in coronavirus disease 2019 outcomes: systematic review and meta-analysis. Chest 2021;159:101940.

3. Fajgenbaum DC, June CH. Cytokine Storm. N Engl J Med 2020;383:2255-73.

4. Tomazini BM, Maia IS, Cavalcanti AB, et al. Effect of Dexamethasone on Days Alive and Ventilator-Free in Patients with Moderate or Severe Acute Respiratory Distress Syndrome and COVID-19: The CoDEX Randomized Clinical Trial. JAMA 2020;324:1307-16.

5. The RECOVERY Collaborative Group. Dexamethasone in hospitalized patients with Covid-19. N Engl J Med 2021;384:693-704.

6. Sterne JAC, Murthy S, Diaz JV, et al. Association between administration of systemic corticosteroids and mortality among critically ill patients with COVID-19: a meta-analysis. JAMA 2020; 324:1330-41.

7. National Institutes of Health. COVID-19 treatment guidelines panel. Coronavirus disease 2019 (COVID-19) treatment guidelines. National Institutes of Health, 2020. Available from: https://www.covid19treatmentguidelines.nih.gov/

8. Keller MJ, Kitsis EA, Arora S, et al. Effect of systemic glucocorticoids on mortality or mechanical ventilation in patients with COVID-19. J Hosp Med 2020;15:489-93.

9. Singh AK, Majumdar S, Singh R, Misra A. Role of corticosteroid in the management of COVID-19: A systemic review and a clinician's perspective. Diabetes Metab Syndr 2020;14:971-8.

10. Bartoletti M, Marconi L, Scudeller L, et al. Efficacy of corticosteroid treatment for hospitalized patients with severe COVID-19: a multicentre study. Clin Microbiol Infect 2021;27:105-11.

11. Tortajada C, Colomer E, Andreu-Ballester JC, et al. Corticosteroids for COVID-19 patients requiring oxygen support? Yes, but not for everyone: Effect of corticosteroids on mortality and intensive care unit admission in patients with COVID-19 according to patients' oxygen requirements. J Med Virol 2021;93:1817-23.

12. Chroboczek T, Lacoste M, Wackenheim C, et al. Corticosteroids in patients with COVID-19: what about the control group? Clin Infect Dis 2021;72:1102-3.

13. Fadel R, Morrison AR, Vahia A, et al. Early short-course corticosteroids in hospitalized patients with COVID-19. Clin Infect Dis 2020;71:2114-20.

14. Jeronimo CMP, Farias MEL, Val FFA, et al. Methylprednisolone as adjunctive therapy for patients hospitalized with coronavirus disease 2019 (COVID-19; Metcovid): a randomized, double-blind, phase iib, placebocontrolled trial. Clin Infect Dis. 2020;72:e373-81.

15. Azienda Unità Sanitaria Locale Reggio Emilia. Patients E of the $\mathrm{E}$ of $\mathrm{HD}$ of $\mathrm{M}$ in S-C( C-19) P. No Evaluation of the Efficacy of High Doses of Methylprednisolone in SARS-CoV2 ( COVID-19) Pneumonia Patients (Unpublished results). Available from: https://clinicaltrials.gov/ct2/show/NCT04673162?term=Meth ylprednisolone \& cond $=$ Covid19\& $\&$ cntry $=\mathrm{IT} \&$ draw $=1 \&$ rank $=1$

16. Confalonieri M, Salton MF. Methylprednisolone vs. dexamethasone in COVID-19 pneumonia (MEDEAS RCT) (Unplublished results). Available from: https://clinicaltrials.gov/ct2/show/NCT04636671?term=Meth ylprednisolone $\&$ cond $=$ Covid $19 \&$ draw $=2$

17. Yadav H. Steroid dosing by bioMARker guided titration in critically ill patients with pneumonia (unpublished results). Available from: https:/clinicaltrials.gov/ct2/show/NCT03852537?term=Meth ylprednisolone \& cond $=$ Covid19\&draw $=3$

18. Russell CD, Millar JE, Baillie JK. Clinical evidence does not support corticosteroid treatment for 2019-nCoV lung injury. Lancet 2020;395:473-5.

19. WHO. Clinical management of COVID-19: interim guidance. WHO, 27 May 2020. Available from: WHO/2019$\mathrm{nCoV} /$ clinical/2020.5:1-62

20. Bellani G, Grasselli G, Cecconi M, et al. Noninvasive ventilatory support of COVID-19 patients outside the intensive care units (WARd-COVID). Ann Am Thorac Soc 2021;18:1020-6.

21. Coppadoro A, Benini A, Fruscio R, et 00611. Helmet CPAP to treat hypoxic pneumonia outside the ICU: an observational study during the COVID-19 outbreak. Crit Care 2021;25:80.

22. Coen D, Paolillo C, Cavazza M, et al. Changing emergency department and hospital organization in response to a changing epidemic. Emerg Care J 2020;16:8969.

23. Chen $\mathrm{G}, \mathrm{Wu} \mathrm{D}$, Guo W, et al. Clinical and immunological features of severe and moderate coronavirus disease 2019. J Clin Invest 2020;130:2620-9.

24. Moore JB, June CH. Cytokine release syndrome in severe COVID-19. Sci 2020;368:473-4.

25. Ahmed MH, Hassan A. Dexamethasone for the treatment of coronavirus disease (COVID-19): a review. SN Compr Clin Med 2020;2:2637-46.

26. Siddiqi HK, Mehra MR. COVID-19 illness in native and immunosuppressed states: A clinical-therapeutic staging proposal. J Heart Lung Transplant 2020;39:405-7.

27. Bahl A, Johnson S, Chen NW. Timing of corticosteroids impacts mortality in hospitalized COVID-19 patients. Intern Emerg Med 2021;16:1593-603.

28. Goleva E, Covar R, Martin RJ, Leung DYM. Corticosteroid pharmacokinetic abnormalities in overweight and obese corticosteroid resistant asthmatics. J Allergy Clin Immunol Pract 2016;4:357-60.e2.

29. Meduri GU, Annane D, Confalonieri M, et al. Pharmacological principles guiding prolonged glucocorticoid treatment in ARDS. Intensive Care Med 2020;46:2284-96.

30. Rafiullah M, Siddiqui K. Corticosteroid use in viral pneumonia: Experience so far and the dexamethasone breakthrough in coronavirus disease-2019. J Comp Eff Res 2020;9:1247-54.

31. Li Y, Meng Q, Rao X, et al. Corticosteroid therapy in critically ill patients with COVID-19: a multicenter, retrospective study. Crit Care 2020;24:698.

32. Shang L, Zhao J, Hu Y, et al. On the use of corticosteroids for 2019-nCoV pneumonia. Lancet 2020;395:683-4.

33. Hashimoto S, Sanui M, Egi M, et al. The clinical practice guideline for the management of ARDS in Japan. J Intensive Care 2017;5:50.

34. Villar J, Ferrando C, Martínez D, et al. Dexamethasone treatment for the acute respiratory distress syndrome: a multicentre, randomised controlled trial. Lancet Respir Med 2020;8:267-76.

35. Meduri GU, Siemieniuk RAC, Ness RA, Seyler SJ. Prolonged low-dose methylprednisolone treatment is highly effective in reducing duration of mechanical ventilation and mortality in patients with ARDS. J Intensive Care 2018;6:53. 
36. Annane D, Pastores SM, Rochwerg B, et al. Correction to: Guidelines for the diagnosis and management of critical illness-related corticosteroid insufficiency (CIRCI) in critically ill patients (Part I): Society of Critical Care Medicine (SCCM) and European Society of Intensive Care Medicine (ESIC. Intensive Care Med 2018;44:474-7.

37. Mueller AA, Tamura T, Crowley CP, et al. Inflammatory biomarker trends predict respiratory decline in COVID-19 patients. Cell Rep Med 2020;1:100144.

38. Hariyanto TI, Japar KV, Kwenandar F, et al. Inflammatory and hematologic markers as predictors of severe outcomes in
COVID-19 infection: A systematic review and meta-analysis. Am J Emerg Med 2021;41:110-9.

39. Ji P, Zhu J, Zhong $\mathrm{Z}$, et al. Association of elevated inflammatory markers and severe COVID-19: A metaanalysis. Med (Baltimore) 2020;99:e23315.

40. Coppo A, Bellani G, Winterton D, et al. Feasibility and physiological effects of prone positioning in non-intubated patients with acute respiratory failure due to COVID-19 (PRON-COVID): a prospective cohort study. Lancet Respir Med 2020;8:765-74. 